This is an author produced version of a paper published in Experimental Hematology. This paper has been peer-reviewed but does not include the final publisher proof-corrections or journal pagination.

Citation for the published paper:

Skold, Stefan and Rosberg, Bodil and Olofsson, Tor

"The N-terminal tetrapeptide of neutrophil proteinase 3

causes S-phase arrest in granulopoietic progenitors."

Exp Hematol. 2005 Nov;33(11):1329-36.

http://dx.doi.org/10.1016/j.exphem.2005.07.015

Access to the published version may require journal subscription.

Published with permission from: Elsevier 


\title{
The N-terminal Tetrapeptide of Neutrophil Proteinase 3 Causes S-phase
}

\section{Arrest in Granulopoietic Progenitors.}

\author{
Stefan Sköld, Bodil Rosberg and Tor Olofsson \\ Division of Hematology \& Transfusion Medicine, Department of Laboratory Medicine, Biomedical Center,
}

Lund University, Lund, Sweden

\begin{abstract}
Objective: Secreted enzymatically inactive proforms of hematopoietic serine proteases proteinase 3 (PR3), azurocidin, and granzymes $\mathrm{A}, \mathrm{B}, \mathrm{H}, \mathrm{K}$, and $\mathrm{M}$, are able to reduce the fraction of granulopoietic progenitors (CFU-GM) in S-phase, whereas human leukocyte elastase (HLE) and cathepsin G lack this ability. The objective of the present study was to map the specific sequence(s) of PR3 and other hematopoietic serine proteases responsible for the downmodulation of S-phase.

Methods: Synthetic peptides corresponding to N-terminal sequences of PR3, purified recombinant PR3 and HLE, as well as hybrid proteins constructed by interchanging the $\mathrm{N}$-terminal regions of PR3 and HLE, thus creating PR3/HLE and HLE/PR3, respectively, were tested for their ability to reduce the fraction of human marrow CFU-GM killed by cytosine arabinoside. In addition, we measured the effect of synthetic peptides on bromodeoxyuridine (BrdU) incorporation in common myeloid progenitors (CMP) and granulocyte/macrophage progenitors (GMP) isolated by cell sorting.

Results: The common N-terminal motif of PR3 and other serine proteases, i.e. IVGG or IIGG, downmodulate the S-phase of CFU-GM at 40-80 nM concentration. Tetrapeptide IVGG, but not IVGR, significantly reduces BrdU-incorporation in GMP within the CD34+ population. When the N-terminal of HLE is presented by the HLE/PR3 hybrid protein it is fully active.

Conclusion: These findings demonstrate that the downmodulatory effect on CFU-GM in S-phase is an Sphase arrest mediated by the first four N-terminal amino acids of PR3, and also suggest that this activity is dependent on the configuration of the proform providing the correct presentation of this $\mathrm{N}$-terminal motif.
\end{abstract}

\section{INTRODUCTION}

Proteinase 3 (PR3), human leukocyte elastase (HLE), cathepsin G and azurocidin are stored in the azurophil granules of the mature neutrophil $(1,2)$. They belong to the hematopoietic serine protease superfamily which also include granzymes A, B, H, $\mathrm{K}$, and $\mathrm{M}$ in cytotoxic T-cells/NK-cells (3), and mast cell chymase (4). They all have a high degree of sequence homology, and share the same principal processing, but the individual proteases also seem to be subjected to subtle differences in this processing $(1,5-7)$. They are synthesized as pre-proenzymes with a signal sequence which is cleaved off immediately after translation/insertion into ER, and an N-terminal dipropeptide, which is cleaved off during processing to give an active enzyme stored in the azurophil granules $(1,5)$. It is generally believed that when the dipropeptide is cleaved off, the new N-terminal of the mature form is turned into the molecule and becomes hidden, at the same time changing the conformation of the protein and making it enzymatically active $(8,9)$. However, a minor portion escapes processing and granule storage, and is instead secreted as proforms with varying length of the propeptide (10). We have identified secreted pro-PR3 as a downregulator of CFU-GM in S-phase (10), and could ascribe the Sphase downregulatory effect almost exclusively to the di-propeptide form (11); thus the effect is not dependent on enzymatic activity. The same S-phase downregulatory effect could also be shown for azurocidin and granzymes A, B, H, K and M (11). On the contrary, HLE and cathepsin G was shown to lack S-phase downregulatory effect $(10,11)$. Taken 
together, these findings suggested that the effect on CFU-GM in S-phase is dependent on $\mathrm{N}$-terminal structures or amino acid motifs that are unique to the proforms of PR3, azurocidin and granzymes, or which are not exposed by the mature forms, respectively.

In the present study we wanted to define the structural determinants of PR3 responsible for Sphase downmodulation, and now demonstrate that the N-terminal tetrapeptide IVGG of mature PR3, or the corresponding IIGG in other serine proteases, have full ability to downmodulate the S-phase of CFU-GM. However, presence of this tetrapeptide motif does not in itself suffice for activity as demonstrated by the inactive proforms of human leukocyte elastase (HLE) or cathepsin G (11), but a HLE/PR3 hybrid protein gainsed activity demonstrating that the HLE N-terminal can excert activity when presented by PR3. In addition, we demonstrate that the mechanism of S-phase downmodulation is an arrest in S-phase as evidenced by a reduced incorporation of the thymidine analog bromodeoxyuridine (BrdU).

\section{MATERIAL AND METHODS}

\section{Synthetic peptides and cytokines}

The following peptides derived from PR3 were synthesized and analyzed by HPLC and mass spectrometry (BioMolecular Resource Facility, University of Lund): peptide\#1: AARAAEIVGGHEA (-6 to +7$)$, peptide\#2: AEIVGGHEAQPH $(-2$ to +10$)$, peptide\#3: IVGGHEAQPH $(+1$ to +10$)$, peptide\#4: GAARAAE (-7 to-1), and peptide\#5: GGTLIHPSFVLT (+30 to $+41)$, with the N-terminal isoleucine in mature PR3 as number +1 . Synthetic peptides corresponding to $\mathrm{N}$-terminal amino acids +1 to +10 of other hematopoietic serine proteases were also synthesized: HLE (IVGGRRARPH), cathepsin G (IIGGRESRPH), azurocidin (IVGGRKARPR), granzyme B (IIGGHEAKPH), and peptide PR3(Q8K) (IVGGHEAKPH), where glutamin is exchanged by lysine as in granzyme $\mathrm{B}$ at the same position.

In addition, the tetrapeptides IVGG $(+1$ to +4 of PR3, azurocodin, and HLE), IIGG $(+1$ to +4 of cathepsin $\mathrm{G}$, and granzymes $\mathrm{A}, \mathrm{B}, \mathrm{H}, \mathrm{K}$, and $\mathrm{M}$ ), and IVGR were synthesized and tested for activity. Peptides were dissolved in water at $10 \mathrm{mM}$ and stored frozen and diluted in McCoy's medium with
$1 \%$ FCS immediately before testing for effect on CFU-GM in S-phase or BrdU-incorporation (see below). Tetrapeptide AcSDKP (Innovagen AB, Lund, Sweden) was dissolved in IMDM with $1 \%$ FSC and stored at $-80^{\circ} \mathrm{C}$ as $1 \mathrm{mM}$ stock solution. Recombinant human macrophage inflammatory protein (hMIP-1 $\alpha$ ), and transforming growth factor (hTGF- $\beta$ ) were purchased from Stem Cell Technologies (Vancouver, BC, Canada).

Recombinant human leukocyte elastase with predetermined $N$-terminals

Recombinant HLE with either an N-terminal dipropeptide $(\mathrm{HLE} / \mathrm{di}+)$ or no propeptide (HLE/mature) was produced mainly as described (11). Briefly, PCR primers were designed with EcoR1 and Xho1 sites to allow insertion of constructs into the pCEP-Pu2 vector for further transfection to the 293EBNA1 cell line (12), and sequences for a 6xHis-tag and an Enterokinase recognition site to allow purification on Ni-NTAcolumns and cleavage by Enterokinase. As template was used a cDNA for human leukocyte elastase in the pCEP4 vector (13), and the following primers: 5'-GAC TTC GAA TTC CAC CAC CAC CAC CAC CAC GAC GAC GAC GAC AAG

ATT GTG GGG GGC CGG CGA GC-3' (upstream primer for HLE/mature), 5'-GAC TTC GAA TTC CAC CAC CAC CAC CAC CAC GAC GAC GAC GAC AAG TCG GAG ATT GTG GGG GGC CGG CGA GC-3'

(upstream primer for $\mathrm{HLE} / \mathrm{di}+$ ), and 5'-GAC TTC CTC GAG TCA GTG GGT CCT GCT GGC C-3’

(downstream primer for both; EcoRI and Xho1 enzyme restriction sites underlined, sequences for $6 \mathrm{xHis}$ tag and enterokinase recognition site in italics, stop codon in bold).

\section{Recombinant PR3/HLE hybrids}

A two-step PCR was utilized for a reciprocal exchange of the first sixteen $\mathrm{N}$-terminal amino acid residues of the di-propeptide forms of PR3 and HLE, i.e the two residues of the di-propeptide and the first fourteen residues of the mature sequence. For each construct pCEP-Pu2/PR3/di+ (11) and pCEP$\mathrm{Pu} 2 / \mathrm{HLE} / \mathrm{di}+$ (as described above) were used as cDNA templates. For construction of HLE/PR3 two parallell reactions were run; reaction $\alpha$ : upstream primer 5'-CCT GCC TGC CAC TGA GGG TTC-3' and downstream primer 5'-CCG CAT CTG CAG GGA GGC CAT GAA GGG CCA CGC GTG GGG C-3' with pCEP-Pu2/HLE/di+ as template, and reaction $\beta$ : upstream primer $5^{\prime}$-ATG GCC TCC 
CTG CAG ATG CGG-3' and downstream primer 5'-GAC TTC CTC GAG CGC TGT GGG AGG GGC GGT TCA-3' with pCEP-Pu2/PR3/di+ as template (Xho1 restriction site underlined and stop codon in bold). The products from reaction $\alpha$ and reaction $\beta$ were purified on agarose gels, and mixed together to be run in a second step of PCR with upstream primer from reaction $\alpha$ and dowstream primer from reaction $\beta$. In a corresponding fashion PR3/HLE was constructed; reaction $\alpha$ with upstream primer same as above, and downstream primer 5'GCG CAG CTG CAG GGA CAC CAT GTA GGG CCG GGA GTG TGG C-3' with pCEP$\mathrm{Pu} 2 / \mathrm{PR} 3 / \mathrm{di}+$ as template; reaction $\beta$ with upstream primer 5'-ATG GTG TCC CTG CAG CTG CGC-3' and downstream primer 5'-GAC TTC CTC GAG TCA GTG GGT CCT GCT GGC C-3' with pCEP$\mathrm{Pu} 2 / \mathrm{HLE} / \mathrm{di}+$ as template. The final two proteinase 3/elastase hybrid constructs PR3/HLE and HLE/PR3, respectively were cut out and ligated into pCEP-Pu2 and transfected to the 293EBNA1 cell line. DNA sequencing was performed to verify the right sequence of the respective constructs.

By chance we detected a mutation in one of the PR3/HLE constructs where glycine number four was exchanged by an arginine, and this construct was taken to transfection also and purification of the recombinant dipeptide proform, named PR3(G4R)/HLE.

Transfection procedures for HEK293EBNA1 cells Wild type HEK293EBNA1 cells (12) were grown in DMEM with $10 \% \mathrm{FCS}$, penicillin $50 \mathrm{IU} / \mathrm{mL}$ streptomycin $50 \mathrm{Ug} / \mathrm{mL}$ and geneticin $250 \mu \mathrm{g} / \mathrm{mL}$. Lipofectin (Superfect, Qiagen $\mathrm{GmbH}$, Germany) was used according to the manufacturer's protocol for stable transfection of adherent cells. After transfection, cells were grown $24-48 \mathrm{~h}$ in DMEM $/ 10 \%$ FCS/geneticin before passage and addition of puromycin $0.5 \mu \mathrm{g} / \mathrm{mL}$ for selection of transfected clones. At confluency, cells were washed twice with PBS and medium was changed to serumfree DMEM with ascorbate $(50 \mu \mathrm{g} / \mathrm{mL})$ for production of recombinant protein. Every second day conditioned medium (CM) was removed and stored frozen until processing, and fresh medium was added for another round of CM production for a total of 3-4 harvests.

Purification of recombinant proteins produced in 293EBNA1 cells
Purification was performed mainly as described (11). Briefly, serum-free CM were pooled, filtered and adjusted to $\mathrm{pH} 7.8,500 \mathrm{mM} \mathrm{NaCl}$ and $10 \mathrm{mM}$ imidazole, before incubation with ProBond Ni-NTA resin (Invitrogen, Paisley, UK) at $+4{ }^{\circ} \mathrm{C}$ over night at constant stirring. After washing recombinant protein was eluted with $20 \mathrm{mM}$ sodium-phosphate-buffer $\mathrm{pH}$ 6.0, $500 \mathrm{mM} \mathrm{NaCl}, 150-300 \mathrm{mM}$ imidazole, before concentration and buffer exchange to EKMax reaction buffer $(50 \mathrm{mM}$ Tris- $\mathrm{HCl} \mathrm{pH} 8.0,1 \mathrm{mM}$ $\mathrm{CaCl}_{2}, \quad 0.1 \%$ Tween-20). Concentrated pure recombinant protein was then subjected to enterokinase cleavage $\left(\mathrm{EKMax}^{\mathrm{TM}}, 1 \mathrm{U} / \mu \mathrm{l}\right.$; Invitrogen) at room temperature over night. Purity and size of cleaved protein was confirmed on SDSPAGE.

\section{S-phase analysis of CFU-GM}

This was done as previously described $(10,11)$. Briefly, human bone marrow mononuclear cells from healthy donors (after informed consent and approved by the Ethical Committe of the University Hospital in Lund) were incubated in duplicates with McCoy's medium 1\% FCS (control) and synthetic peptides or purified recombinant proteins for $90 \mathrm{~min}$ at $37^{\circ} \mathrm{C}$. After $90 \mathrm{~min} 2 \mathrm{ug} / \mathrm{mL}$ cytosine arabinoside (Cytosar, Pharmacia \& Upjohn) was added to one of the tubes in each pair and the incubation continued for another $45 \mathrm{~min}$ to kill cells in S-phase. Cells were then washed and cultured in agar in four replicates with $20 \mathrm{ng} / \mathrm{mL}$ G-CSF (Neupogen, Roche) and $20 \mathrm{ng} / \mathrm{mL}$ GM-CSF (Leucomax, ScheringPlough) as colony-stimulating factors. CFU-GM colonies of more than 50 cells were counted on day 11. The reduction in colony number after incubation with cytosine arabinoside is a measure of the number of CFU-GM in S-phase. Colony numbers were always $>100 /$ dish. In some experiments CD34+ cells were isolated by magnetic microbeads (CD34 progenitor cell isolation kit; Miltenyi Biotec, Bergisch Galdbach, Germany) and used as target cells as described (10); the purity of the CD34+ cells used in these experiments was more than $90 \%$.

Bromodeoxyuridine (BrdU) incorporation in progenitor cells

As another means of cell cycle analysis we measured BrdU incorporation in progenitor cells isolated by cell sorting. BrdU incorporation was measured using the Absolute-S Kit (Phoenix Flow Systems, San Diego, CA) and all steps were performed as described by the manufacturer. Briefly, 
human CD34+ marrow cells isolated by immunomagnetic beads (Miltenyi Biotec) were incubated with tetrapeptides IVGG or IVGR (inactive control) $(80 \mathrm{nM})$ for $90 \mathrm{~min}$ at $37^{\circ} \mathrm{C}$ before addition of BrdU for another $20 \mathrm{~min}$. Thereafter cells were washed twice in cold medium and labeled with monoclonal antibodies. The following combinations were used: CD45RA-PE/CD19PerCP-Cy5.5/CD34-APC (without any FITC-labeled MoAb), CD19-FITC/CD123-PE/CD34-PerCPCy5.5/CD45RA-APC, and CD34-FITC/CD123PE/CD19-PerCP-Cy5.5/CD45RA-APC (all from BD Biosciences, San Jose, CA) and sorted on a FACS Aria cell sorter (BD Biosciences). CD34+/CD19cells were sorted into CD45RA- and CD45RA+ subpopulations representing common myeloid progenitors (CMP) and granulcoyte/macrophage progenitors (GMP), respectively (14). 100-200.000 cells of each population was collected into cold medium and directly taken to further processing with Photolyte Enhancer, fixation in ice-cold $70 \%(\mathrm{v} / \mathrm{v})$ ethanol and storage at $-20^{\circ} \mathrm{C}$ for at least 18 hours before proceeding with UV-light treatment $(8 \mathrm{~min})$, BrdUTP incorporation and staining with anti-BrdUFITC antibody. After dilution in buffer containing PI (propidium iodide) and RNase, BrdU-incorporation was measured by flow cytometry (FACScan, BD Biosciences) within $30 \mathrm{~min}$. Cell debris and doublets were excluded from analysis by gating on PI in a flourescence area versus width dot plot. Cell cycle distribution based on the incorporation of PI was analyzed using ModFit LT software (Verity Software House, Topsham, ME).

\section{RESULTS}

Synthetic peptides of PR3 and other serine proteases Four different peptides corresponding to N-terminal sequences of PR3 were synthetically produced and taken to assay for CFU-GM in S-phase. Peptides \#1, $\# 2$, and \#3, downmodulated the S-phase fraction of CFU-GM at $20 \mathrm{nM}$ (Fig.1). Downmodulation was dose-dependent over a range of $0.8,4,20$, and 100 $\mathrm{nM}$, with a tendency towards a biphasic response with a slightly weaker or equal inhibitory activity at $100 \mathrm{nM}$ as at $20 \mathrm{nM}$ (data not shown). Peptides \#1\#3 all contain the initial seven amino acids of the mature form of PR3. Peptide \#4, which represents the seven amino acids of the propeptide preceeding the isoleucine of mature PR3, and peptide \#5, corresponding to amino acids $30-41$ of mature PR3 were both inactive (0.8-100 $\mathrm{nM})$. These findings show that the propeptide amino acids are dispensable for activity and that capacity to downmodulate CFU-GM in S-phase is exerted by peptides which share the common IVGGHEA motif of mature PR3.

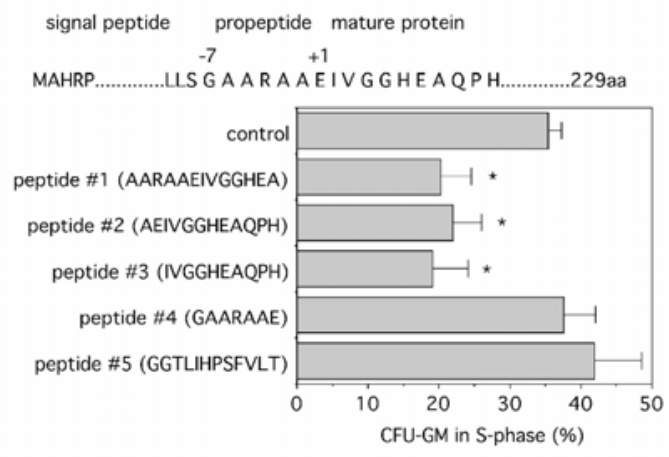

Fig.1. Reduction of the fraction of CFU-GM in Sphase by synthetic peptides corresponding to different parts of the N-terminal region of PR3 (peptides \#1-\#4) and an internal sequence corresponding to amino acids 30-41 (peptide \#5). The peptide sequences are shown within brackets. Peptides were tested at $20 \mathrm{nM}$ final concentration. Results are mean values of 3-4 experiments and the bars show $\mathrm{SD} ; *$ denotes significant difference from the control $(\mathrm{p}<0.01)$.

Since the N-terminal sequences of all hematopoietic serine proteases are similar synthetic peptides corresponding to the first 10 amino acids of mature azurocidin, HLE, cathepsin G and granzyme B, respectively, were tested for activity in comparison with the PR3 peptide equivalent, i.e. peptide \#3. However, only PR3 peptide \#3 was able to downmodulate the S-phase of CFU-GM (Fig.2). Interestingly, if glutamin in position eight is substituted with lysine (Q8K), as in granzyme B, the resulting peptide becomes inactive just as the granzyme B peptide. These results demonstrate that although the peptides share the common IVGG/IIGG motif the composition of the following six amino acids affects the activity. 


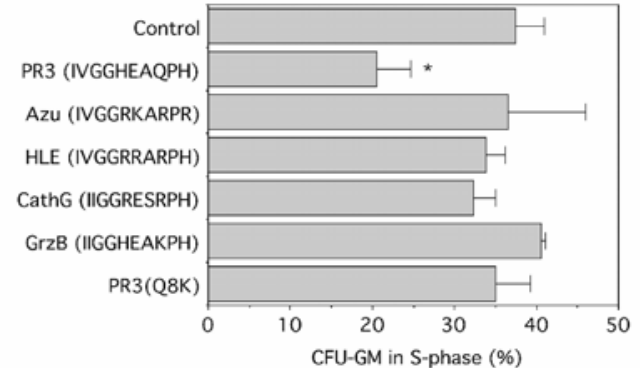

Fig.2. Comparison of the S-phase down modulatory effect of synthetic peptides corresponding to the first ten amino acids of mature PR3, azurocidin (Azu), human leukocyte elastase (HLE), cathepsin G (CathG), granzyme B (GrzB), and a mutated form of the PR3 peptide (PR3/8QK). The peptide sequences are shown within brackets. The peptides were tested at 20 and $100 \mathrm{nM}$ concentration and the results from $20 \mathrm{nM}$ are shown; the higher concentration did not change the overall results. Results are mean values of three experiments and the bars show SD; * denotes significant difference from the control $(p<0.01)$. PR3 $(Q 8 K)$ is significantly different from unmutated PR3 $(\mathrm{p}<0.01)$.

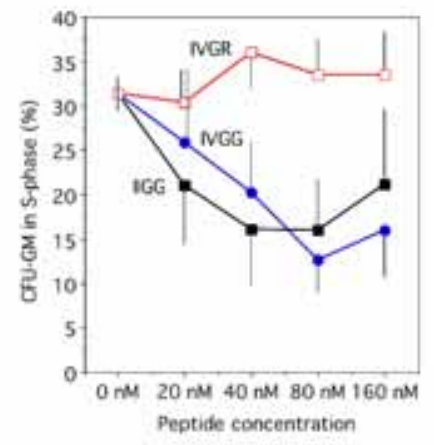

Fig.3. CFU-GM S-phase downmodulation mediated by tetrapeptides IVGG (closed circles), IIGG (closed boxes), and IVGR (open boxes). Results are mean values of four different experiments and the bars show SD. IVGG caused significant downmodulation at 40,80 , and $160 \mathrm{nM}$, and IIGG at 40 and $80 \mathrm{nM}$, respectively $(p<0.01)$. IVGR had insignificant effects.
In the next set of experiments we tested whether the common tetrapeptide motifs IVGG/IIGG by themselves were active. As shown in Fig.3 both tetrapeptides significantly downmodulated the Sphase fraction in a dose-dependent manner with maximum effect at $40-80 \mathrm{nM}$, which is approximately 10-20 times higher concentration than required for native pro-PR3 (10). Tetrapeptide IVGR was inactive.

Interchange of $N$-terminal sequences between PR3 and HLE

The proform of HLE does not exhibit S-phase downmodulatory activity despite the presence of the active tetrapeptide motif IVGG (11). To test the hypothesis that the N-terminal of HLE is incorrectly presented to execute this activity, we exchanged the $\mathrm{N}$-terminal region of PR3 with the $\mathrm{N}$-terminal region of HLE including the di-propeptide (HLE/PR3) (Fig.4). In addition, we produced the reversed transfer and exhanged the N-terminal of HLE with the N-terminal region of PR3 (PR3/HLE) (Fig.4) to test whether the $\mathrm{N}$-terminal of PR3 becomes inactive when presented by HLE. If the hypothesis was correct the HLE/PR3 fusion protein would be active, which turned out to be the case as shown in Fig.5. This finding demonstrates that the $\mathrm{N}$-terminal of HLE gain activity if coupled to the C-terminal part of PR3. However, the reciprocal N-terminal exchange, i.e. PR3/HLE, also was active (Fig.5) but less potent than the native pro-PR3. The mutant form of PR3/HLE, however, with an exchange of glycine number four by arginine, i.e. PR3(G4R)/HLE, lost all activity, which underlines the importance of the IVGG/IIGG motif. This was further substantiated by the observation that when the peptide IVGG is changed to IVGR it becomes totally inactive (Fig.3).

AcSDKP, MIP- $1 \alpha$ and TGF- $\beta$

For comparison some other known inhibitors of hematopoietic stem and progenitor cell proliferation were tested in the same way as PR3 derived peptides. AcSDKP at 50 or $100 \mathrm{ng} / \mathrm{mL}$ did not reduce the fraction of CFU-GM killed by cytosine arabinoside (control: $34,1 \% \pm 4$; AcSDKP 50 $\mathrm{ng} / \mathrm{mL}: 36,6 \% \pm 1,0 ;$ AcSDKP $100 \mathrm{ng} / \mathrm{mL}: 33,4 \% \pm$ $2,7 ; \mathrm{n}=4)$. MIP- $1 \alpha$ at 50 and $100 \mathrm{ng} / \mathrm{mL}$ was also without effect $(50 \mathrm{ng} / \mathrm{mL}: 35,5 \% \pm 4,0 ; 100 \mathrm{ng} / \mathrm{mL}$ : $35,7 \% \pm 2,6 ; \mathrm{n}=4)$, just as TGF- $\beta$ at $25 \mathrm{ng} / \mathrm{mL}$ $(36,6 \% \pm 7,1 ; n=3)$. 


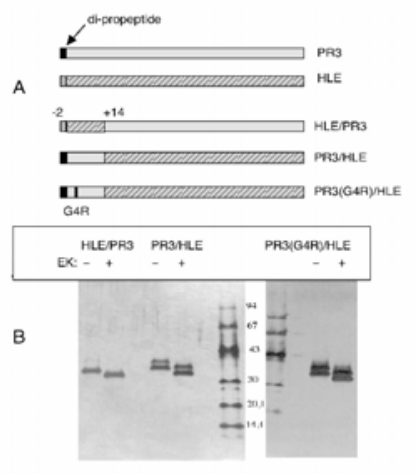

Fig.4. Panel A. Schematic outline of PR3, HLE, and the hybrids produced in recombinant form. Panel B. The electrophoretic pattern on SDS-PAGE and silver staining of the purified fusion proteins HLE/PR3, PR3/HLE, and the mutated PR3(G4R)/HLE, respectively, before and after removal of the His-tag by enterokinase (EK) cleavage. The hybrids containing a HLE C-terminal show two bands, which is identical with the electrophoretic pattern for recombinant wild type HLE (data not shown). This two-band pattern is probably due to differences in glycosylation or incomplete $\mathrm{C}$-terminal processing that removes the 20 amino acid C-terminal prodomain, which is not necessary for enzymatic activity nor for targeting to granules (13). Both components contain the His-tag as evidenced by the shift in mobility after enterokinase treatment.

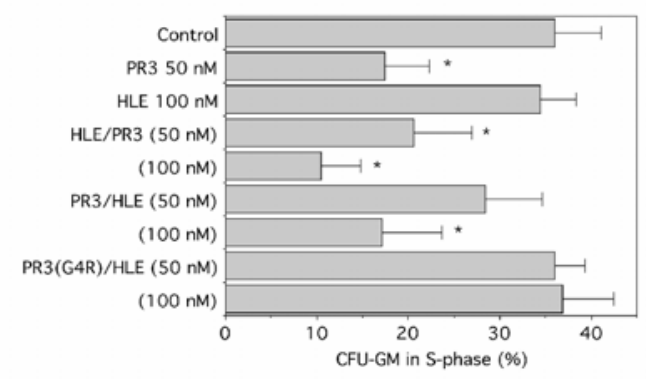

Fig.5. Reduction of the S-phase fraction of CFUGM by recombinant dipeptide proforms of wild type PR3 and HLE, the two reciprocal N-terminal switches HLE/PR3 and PR3/HLE, and the mutant PR3(G4R)/HLE, respectively. Results are mean values of three experiments and the bars show SD. * denotes significant difference from the control $(\mathrm{p}<0.05)$.
PR3 peptides act on purified CD34+ cells Previously we have shown that the proform of PR3 acts directly on target cells within the CD34+ hematopoietic progenitor population (10). To test whether this is true also for the inhibitory PR3 peptides, peptides \#1 -3 and the tetrapeptides IVGG/IIGG were tested for activity towards purified human marrow $\mathrm{CD} 34+$ progenitor cells. As shown in Fig.6 the peptides also were active towards purified $\mathrm{CD} 34+$ cells suggesting that they act directly on the progenitor cells rather than via indirect mechanisms.

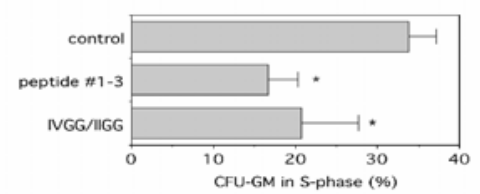

Fig.6. Peptides act directly on CD34+ cells. Isolated bone marrow CD34+ cells were used as target cells for peptides \#1-3 (20 nM) and tetrapeptides IVGG and IIGG $(80 \mathrm{nM})$. Columns show mean values and bars SD; controls $(\mathrm{n}=6)$, peptide \#1 $(\mathrm{n}=3)$, peptide \#2 $(n=2)$, peptide \#3 $(n=4)$, IVGG $(n=2)$, and IIGG $(\mathrm{n}=2)$. For statistical testing peptides $\# 1-3$, and IVGG/IIVV, respectively, were grouped together; * denotes significant difference from the controls (peptides \#1-3: p-value 0.001, IVGG/IIGG: p-value $0.011)$.

PR3 peptides inhibit BrdU incorporation in granulocyte/macrophage progenitor cells (GMP)

Fig.7 shows the isolation of CMP and GMP populations by cell sorting according to the definitions proposed by Manz et al (14). CD19+ cells were excluded as the major non-myeloid component of marrow CD34+ cells and CD34+/CD19- cells were divided into CD1231o/CD45RA- (CMP) and CD123lo/CD45RA+ (GMP), respectively, or CD45RA- and CD45RA+ in those experiments CD123 was not included. However, the important discriminator between CMP and GMP is CD45RA. An example of BrdU incorporation by GMP is shown in Fig.7 (lower part). The collective data of nine separate marrow samples demonstrated that 
IVGG significantly inhibited the BrdU incorporation of GMP but hardly so of CMP, whereas full-length proPR3 inhibited BrdU incorporation of both progenitor populations (Fig.8). However, when the cell cycle distribution according to PI-labeling was analyzed no significant differences were observed between controls and IVGG-exposed cells (data not shown).

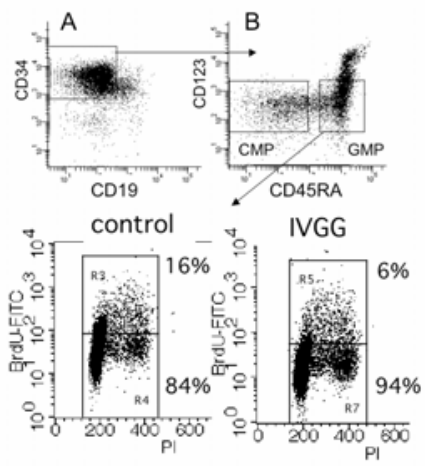

Fig.7. IVGG inhibits BrdU incorporation in granulocyte/macrophage progenitors. Panels A and $\mathrm{B}$ show the isolation of CMP and GMP populations by flourescence activated cell sorting.

CD34+/CD19- cells were divided into

CD1231o/CD45RA- (CMP) and

CD123lo/CD45RA+ (GMP). The two lower panels show an example of BrdU incorporation in GMP; $16 \%$ of the control cells incubated with the inactive IVGR peptide $(80 \mathrm{nM})$ incorporated $B r d U$, whereas only $6 \%$ of the cells incubated with IVGG $(80 \mathrm{nM})$ incorporated BrdU, using the upper limit of the $\mathrm{G} 2 / \mathrm{M}$ population as cut off. BrdU-FITC on $\mathrm{Y}$-axis and PI on the $\mathrm{X}$-axis.

\section{DISCUSSION}

Previously we have shown that the capacity of PR3 to downmodulate the S-phase of granulopoietic progenitors is restricted to the dipeptide-containing proform, and the same is true for azurocidin and granzyme B, whereas the mature enzymatically active forms lack S-phase regulatory activity (11). In the proform conformation of hematopoietic serine proteases the N-terminal is exposed on the surface of the molecule as recently confirmed by the crystal structure of pro-granzyme K (15). Removal of the dipeptide results in conformational changes and the $\mathrm{N}$-terminal is turned inwards and is no longer exposed on the surface of the mature protein $(8,9)$. Therefore, we have argued that the activity towards granulopoietic progenitors probably is exerted by $\mathrm{N}$ - terminal structures. The initial tests of synthetic peptides derived from N-terminal sequences of PR3 proved that this assumption was correct and that the activity resided within the sequence IVGGHEA common to the active peptides. However, the finding that the N-terminal decapeptide of granzyme B was inactive was unsuspected in view of the fact that the full length pro-granzyme $B$ is active. The observation that a single amino acid exchange in the PR3 decapeptide (Q8K) abrogated the activity, suggested that the activity of the decapaptides are influenced by net charge of the molecules. For instance, the inactive PR3(Q8K) peptide gains another basic amino acid making its net charge equal to the inactive granzyme $\mathrm{B}$ and cathepsin $\mathrm{G}$ decapeptides, respectively, which supports this interpretation.

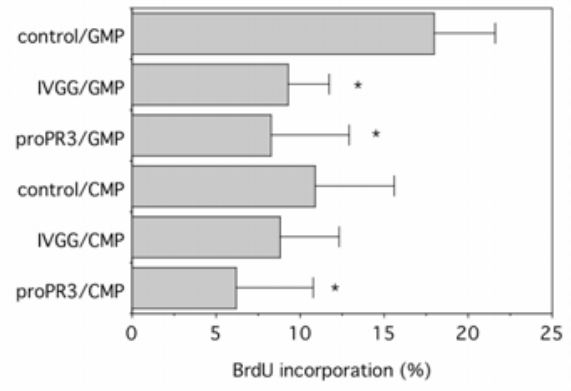

Fig.8. BrdU incorporation in purified GMP and CMP populations. IVGG $(80 \mathrm{nM})$ inhbited BrdU incorporation in GMP cells $(p=0,001 ; n=9)$ but not signifcantly in CMP cells $(\mathrm{p}=0,052 ; n=9)$. ProPR3 $(50 \mathrm{ng} / \mathrm{mL})$ inhibited BrdU incorporation in both $\operatorname{GMP}(\mathrm{p}=0,001 ; \mathrm{n}=4)$ and CMP $(\mathrm{p}=0,027 ; \mathrm{n}=4)$. Control cells were incubated with IVGR $(80 \mathrm{nM})$. Cell sorting and BrdU incorporation was measured as outlined in Fig.7. Results are mean values and bars show $\mathrm{SD} ; *$ denotes significant difference from the respective control by Student's paired t-test.

Next we suspected that the activity may be executed by the most N-terminal amino acids IVGG. These are the first four amino acids of mature PR3, azurocidin, and elastase, whereas cathepsin $\mathrm{G}$ and all the granzymes start with IIGG. In fact, both these tetrapeptides were active towards granulopoietic progenitors, and a single amino acid change from IVGG to IVGR totally abrogated the effect. Thus it is evident that the IVGG/IIGG motif mediates the S- 
phase downmodulatory signal. However, it is also evident that the presence of this amino acid motif is not in itself enough for activity when it comes to the native proteases. Presentation or conformation of the $\mathrm{N}$-terminal region is also important for activity as demonstrated by switching the N-terminals between PR3 and HLE. The HLE/PR3 fusion protein demonstrated that the HLE N-terminal gains activity when fused to PR3 and suggests that the presentation of the N-terminal of HLE is different from that of wild type HLE. On the other hand, the PR3/HLE fusion protein retained activity, but was less potent than wild type pro-PR3 or HLE/PR3, which could be explained by a less efficient presentation of the N-terminal of PR3 by HLE than by wild type PR3. In both cases, the fusion proteins demonstrate the importance of the overall conformation of the fusion proteins as well as the wild type proforms for the activity towards CFUGM. However, the IVGG/IIGG motif must be intact as demonstrated by the mutated PR3(G4R)/HLE which had no activity, as well as the inactive tetrapeptide IVGR.

The peptides were active towards purified CD34+ marrow cells and furthermore, inhibited incorporation of $\mathrm{BrdU}$ in granulocyte/macrophage progenitors (GMP) without changing the cell cycle distribution, which taken together indicate that the peptides cause an arrest in S-phase. This observation explains the mechanism and rapid onset of the Sphase downmodulatory effect of pro-PR3 and the Nterminal peptides shown here. By arresting progenitor cells in S-phase pro-PR3 reduces not only incorporation of $\mathrm{BrdU}$ but also of cytosine arabinoside leading to reduced killing of cells in Sphase as measured in the colony assay.

Previously described inhibitors such as the tetrapeptide AcSDKP (acetyl-N-Ser-Asp-Lys-Pro) and chemokine MIP-1 $\alpha$ inhibits the cycling of normal progenitors (16-19) but did not reduce the killing of CFU-GM by cytosine arabinoside in the short term assay used in the present investigation. TGF- $\beta$, another inhibitor of progenitor cell growth $(18,20)$, was also without effect. These inhibitors generally require longer incubation periods than used in the present study to show inhibition, and the results presented here suggest that the PR3 derived peptides act by mechanisms different from those of AcSDKP, MIP- $1 \alpha$ and TGF- $\beta$, respectively.

\section{Acknowledgement.}

This study was supported by the Swedish Cancer Foundation, Ingabritt and Arne Lundberg's Research Foundation, Georg Danielsson's Foundation, Alfred Österlund's Foundation, John Persson's Foundation, and the Research Funds of the University Hospital in Lund.

\section{References}

1. Gullberg U, Andersson E, Garwicz D, Lindmark A, Olsson I. Biosynthesis, processing, and sorting of neutrophil proteins - insight into neutrophil granule development. Eur J Haematol. 1997;58:137-153.

2. van der Geld YM, Limburg, PC, Kallenberg, CGM. Proteinase 3, Wegener's autoantigen: from gene to antigen. J Leukoc Biol. 2001;69:177-190.

3. Trapani JA. Granzymes: a family of lymphocyte granule serine proteases. Genome Biology 2001; 2:3014.1-3014.7

4. Caughey GH, Schaumberg TH, Zerweck $\mathrm{EH}$, et al. The human mast cell chymase gene (CMA1): mapping to the cathepsin $\mathrm{G} /$ granzyme gene cluster and lineage- restricted expression. Genomics 1993;15:614-620.

5. McGuire MJ, Lipsky PE, Thiele DL. Generation of active myeloid and lymphoid granule serine proteases requires processing by the granule thiol protease peptidase I. J Biol Chem. 1993;268:24582467.

6. Rao NV, Rao GV, Marshall BC, Hoidal JR. Biosynthesis and processing of proteinase 3 in U937 cells. Processing pathways are distinct from those of cathepsin G. J Biol Chem. 1996;271:2972-2978

7. Lindmark A, Garwicz D, Rasmussen PB, Flodgaard H, Gullberg U. Characterization of the biosynthesis, processing, and sorting of human HBP/CAP37/azurocidin. J Leukoc Biol.1999;66:634-643. 
8. Murakami M, Karnik SS, Husain A. Human prochymase activation. A novel role for heparin in zymogen processing. J Biol Chem. 1995;270: 2218-2223.

9. Fujinaga M, Chernaia MM, Halenbeck R, Koths K, James MN. The crystal structure of PR3, a neutrophil serine proteinase antigen of Wegener's granulomatosis antibodies. J Mol Biol. 1996;261: 267-278.

10. Sköld S, Rosberg B, Gullberg U, Olofsson T. A secreted proform of neutrophil proteinase 3 regulates the proliferation of granulopoietic progenitor cells. Blood. 1999;93:849-856.

11. Sköld S, Zeberg L, Gullberg U, Olofsson T. Functional dissociation between proforms and mature forms of proteinase 3 , azurocidin, and granzyme B in regulation of granulopoiesis. Exp Hematol. 2002;30:689-696.

12. Hallgren J, Karlson U, Poorafshar M, Hellman L, Pejler G. Mechanism for activation of mouse mast cell tryptase: dependence on heparin and acidic $\mathrm{pH}$ for formation of active tetramers of mouse mast cell protease 6 . Biochemistry 2000;39:13068-13077.

13. Gullberg U, Lindmark A, Lindgren G, Persson AM, Nilsson E, Olsson I. Carboxyl-terminal prodomain-deleted human leukocyte elastase and cathepsin G are efficiently targeted to granules and activated in the rat basophilic/mast cell line RBL. J Biol Chem. 1995;270:12912-12918.

14. Manz MG, Miyamoto T, Akashi K, Weissman IL. Prospective isolation of human clonogenic common myeloid progenitors. Proc Natl Acad Sci USA 2002;99.11872-11877.
15. Hink-Schauer C, Estebanez-Perpiná E, Wilharm $\mathrm{E}$ et al. The 2.2- $\AA$ crystal structure of human pro-granzyme $\mathrm{K}$ reveals a rigid zymogen with unusual features. J Biol Chem. 2002;277:50923-50933.

16. Bonnet D, Lemoine FM, Pontvert-Delucq S, Baillou C, Najman C, Guigon M. Direct and reversible inhibotory effect of the tetrapeptide acetyl-N-Ser-Asp-Lys-Pro (Seraspenide) on the growth of human CD34+ subpopulations in response to growth factors. Blood 1993;82:3307-3314.

17. Cashman JD, Eaves AC, Eaves CJ. The tetrapeptide AcSDKP specifically blocks the cycling of promitive normal but not leukemic progenitors in long-term culture: evidence for an indirect effect. Blood 1994;84:1534-1542.

18. Cashman JD, Eaves CJ, Sarris AH, Eaves AC. MCP-1, not MIP-1 $\alpha$, is the endogenous chemokine that cooperates with TGF- $\beta$ to inhibit the cycling of primitive normal but not leukemic (CML) progenitors in long-term human marrow cultures. Blood 1998;92:2338-2344.

19. Broxmeyer HE, Sherry B, Cooper S, et al. Comparative analysis of the human macrophage inflammatory protein family of cytokines (chemokines) on proliferation of human myeloid progenitor cells. J Immunol. 1993;150:3448-3458.

20. Cashman JD, Eaves AC, Raines EW, Ross R, Eaves CJ. Mechanisms that regulate the cell cycle status of very primitive hematopoietic cells in long-term human marrow cultures. I. Stimulatory role of a variety of mesenchymal cell activators and inhibitory role of TGF- $\beta$. Blood 1990;75:96-101. 